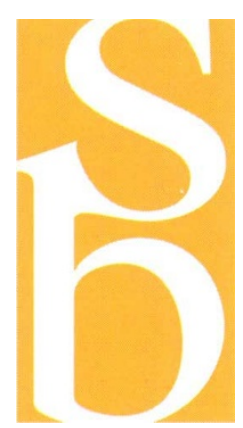

\section{Challenging the predictors}

Those who break their heads trying to predict the fold of a protein or nucleic acid may at least comfort themselves that it is even harder to predict the outcome of NATO air strikes against the Bosnian Serbs, next week's weather or the fluctuation of the currency markets. Such events involve many variables - some of which exhibit chaotic behaviour - and are so fraught with uncertainty that suggested scenarios are unlikely to be anything more than inspired guess work, at best. Is accurate prediction of the secondary/ tertiary fold of an RNA, or protein, structural biology's insoluble problem, or does it have such prominence because it will soon cease to be a problem?

Much attention has been focused on modelling the folded conformation of proteins of late, almost as a matter of necessity: the number of protein sequences determined is far outstripping the number of known protein structures. Indeed there are many algorithms available for predicting the structure of proteins from their amino-acid sequences.

As with most attempts at prediction the accuracy of the final model depends on the available starting information. With sequence data alone one might expect to have a reasonable chance of predicting the localized secondary structure, but there is likely to be little insight concerning the long-range interactions required to define the tertiary conformation. The existence of the structure of a homologous protein will allow the prediction of the tertiary structure with a fair degree of certainty. But which of the numerous algorithms are the most effective, given the various possible starting conditions, and what routes are worth pursuing when striving to improve the accuracy of prediction?
In an attempt to address these questions a prediction contest on a previously unprecedented scale is being organized by John Moult and Jan Pedersen (Centre for Advanced Research in Biotechnology, University of Maryland) Krzytof Fidelis (Lawrence Livermore National Laboratory, California) and Richard Judson (Sandia National Laboratory, California). The organizers at present have, or will have, seventeen unpublished target structures against which hopeful predictors can test their protocols. Prediction methods are divided into three categories: comparative modelling, fold recognition ('threading'), and $a b$ initio prediction. Independent assessors will judge the submitted predictions using standard sets of criteria for the different categories.

Deadlines for predictions of the various proteins will depend on the publication dates of the papers reporting the relevant structures. The challenge is to culminate in a meeting on 4-8 December 1994 in Asilomar, California, where the results of the contest will be announced and discussed (further details of the competition can be obtained from John Moult: tel 301738 6241; fax 301738 6255; internetjmoult@iris4.carb.nist.gov).

A competition such as this should (hopefully) provide a thorough and objective test of the strengths and weaknesses of the prediction algorithms presently available. But much depends on the willingness of the predictors to tackle as many of the target structures as they can, so that all can judge how robust their prediction strategies are.

Other smaller-scale prediction competitions are being announced all the time. A protein-docking prediction challenge has been facilitated by the recent determination of the structure of a $\beta$ lactamase-inhibitor protein (BLIP) by Michael James and colleagues ${ }^{1}$. The structure of the $\beta$-lactamase/BLIP complex is nearing 
completion and a tentative deadline (31 August) for submissions has been set. An algorithm from Abagyan and colleagues, which recently featured in these pages ${ }^{2}$, is among those being used to tackle the problem. Docking two proteins is certainly a challenge; the ability of present protocols to predict the binding modes of small ligands is still far from perfect, as researchers around the world attempting to design drugs that are effective in the treatment of diseases such as AIDS, cancer, malaria, arthritis and so on, are all too aware.

\section{It's an RNA world}

The theme of prediction and modelling is continued in one of a clutch of papers in this issue which deal with RNA structure and function $^{3-5}$. The HIV-1 Rev protein is involved in the control of viral replication and infectivity, and these functions are mediated in part by the interaction of Rev with a Revbinding element $(R B E)$ in viral RNA. To understand further the interaction between protein and nucleic acid, Leclerc and colleagues use the structural constraints identified from artificial phylogenies of Revbinding RNAs generated by in vitro selection together with an RNA modelling program to generate a three-dimensional model of $R B E$. The authors note that the major groove of the $R B E$ 'stem-loop' structure is wider than the standard A-form RNA major groove and can accommodate a potentially $\alpha$-helical region of Rev implicated in $R B E$ binding.

The paper from Majerfield and Yarus ${ }^{4}$ and one recently published elsewhere ${ }^{6}$ use in vitro selection to develop RNAs that bind small ligands with high affinity and specificity: Lvaline, an aliphatic hydrophobe, and theophylline, a clinically important small molecule used in the treatment of asthma, bronchitis, and emphysema. One of the RNAs selected to bind theophylline shows a level of discrimination between the target molecule and the very closely related caffeine molecule which is an order of magnitude better than that for available antibodies ${ }^{6}$.
Will in vitro-selected RNAs compete with antibodies as ligand or epitope-specific reagents, or even catalysts? The increasing repertoire of RNA-mediated interactions and the speed with which such RNAs can be generated, both of which seem to be limited only by the perseverance of the experimenter, make them an attractive proposition as antibody rivals in certain circumstances.

\section{Insight}

This month's issue of Nature Structural Biology contains the first paper to appear in our occasional 'Insight' section. Insight pieces will describe the development of tools, techniques and methodologies intended to be of practical use and interest to those involved in determining the structure and function of biological macromolecules.

Contributions to this section of the journal should conform to the same format as papers which appear in the back half of the journal; in particular, they are subjected to the same rigorous peer review process as Correspondences and Articles which appear in Nature Structural Biology.

The first Insight article provides an apposite example of the kind of material to be considered for publication in this section. Tom Cech and colleagues ${ }^{5}$, continuing the theme of RNA structure and function, now present a new secondary structure presentation for the Michel-Westof 3D model group I introns. This aims to improve on the representation of tertiary structural elements compared with the present 'industrial standard'.

1. Strynadka, N.C.J. et al. Nature 368 , 657-660 (1994)

2. Totrov, M. \& Abagyan, R. Nature struct. Biol. 1, 259-263 (1994)

3. Leclerc, F., Cedergren, R. \& Ellington, A.D. Nature struct. Biol. 1, 293-300 (1994)

4. Majerfeld, I. \& Yarus, M. Nature struct. Biol. 1, 287-292 (1994)

5. Cech, T.R., Damberger, S.H. \& Gutell, R.R. Nature struct. Biol. 1, 273280 (1994)

6. Jenison, R.D., Gill, S.C., Pardi, and Polisky, B. Science 263, 1425-1429

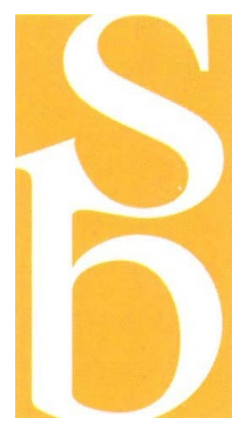

